\title{
Électorat « du centre » et modération politique en Bretagne sous la IIIe République : l'exemple du Finistère et du Morbihan
}

\section{Laurent Paubert}

\section{OpenEdition Journals}

\section{Édition électronique}

URL : http://journals.openedition.org/abpo/1303

DOI : $10.4000 / a b p o .1303$

ISBN : 978-2-7535-1493-5

ISSN : 2108-6443

Éditeur

Presses universitaires de Rennes

Édition imprimée

Date de publication : 20 mars 2004

Pagination : 65-90

ISBN : 978-2-86847-976-1

ISSN : 0399-0826

\section{Référence électronique}

Laurent Paubert, «Électorat « du centre » et modération politique en Bretagne sous la IIIe République l'exemple du Finistère et du Morbihan ", Annales de Bretagne et des Pays de l'Ouest [En ligne],

111-1 | 2004, mis en ligne le 20 mars 2006, consulté le 19 avril 2019. URL : http:// journals.openedition.org/abpo/1303; DOI : 10.4000/abpo.1303 


\title{
Électorat "du centre " et modération politique en Bretagne sous la III République : l'exemple du Finistère et du Morbihan
}

\author{
Laurent PAUBERT \\ Docteur en histoire \\ Université de Brest
}

Lorsque l'on analyse précisément les résultats électoraux des débuts de la Troisième République dans le Finistère et le Morbihan, on réalise rapidement que la réputation de modération qui s'attache à la Bretagne n'est pas usurpée. Pourtant, cette réalité du débat politique breton n'est pas la première qui vienne à l'esprit. "Gwen ar ru " : dans ce face-à-face réducteur semblent se résumer toutes les oppositions politiques de la Bretagne de la fin du dix-neuvième et du début du vingtième siècle. La lutte religieuse relancée par le Bloc des Gauches semble accentuer encore le caractère bipolaire de la vie politique. En Bretagne, on peut cependant isoler, et ceci très précocement, un électorat qui ne s'est jamais retrouvé dans l'antagonisme droite-gauche auquel tend naturellement le mode de scrutin majoritaire. D'importance variable, cet électorat " central ", fluctuant en apparence entre droite et gauche car n'appartenant ni à l'une ni à l'autre, possédait ses logiques et ses motivations propres. Longtemps privé d'expression, il n'en a pas moins toujours existé et a été un élément capital de la vie politique. Dans une première partie, nous verrons que l'existence d'une sensibilité modérée dans l'électorat a existé dès les premières années de la Troisième République. Nous montrerons ensuite que son importance a conduit les politiques à rechercher, par delà le face-à-face gauche-droite, une alliance des modérés des deux camps. C'est au niveau des élections sénatoriales que cette conjonction des centres est le plus aisément remarquable. Une troisième partie permettra d'insister sur les comportements de cet électorat modéré au début du vingtième siècle. Une quatrième partie montrera que, arbitre bien souvent des majorités, cet électorat a fini par imposer ses règles au jeu politique et a amené les candidats à se battre sur son terrain. 


\section{Poids de l'électorat modéré au début de la Troisième République}

Malgré des dissidences plus ou moins organisées, deux blocs se sont électoralement très tôt faits face, dès la Seconde République en fait. Le Second Empire a permis, en même temps qu'une familiarisation avec le vote, de poser les grands thèmes du débat politique pour les quarante ans qui lui succédèrent. La campagne des législatives de 1869 l'illustre à merveille et nombre de lignes de clivages durables s'y remarquent déjà : question sociale et ouvrière, question romaine, défense religieuse et anticléricalisme, question scolaire, conservatisme et monde moderne... Tirant parti de la libéralisation du régime après 1868, la presse avait permis d'exposer et de débattre des grandes questions et orientations politiques. Après vingt ans d'accoutumance au vote, en dépit des modes autoritaires de mobilisation électorale alors en usage, la Bretagne peu à peu entrait dans la modernité politique. Dès la fin de l'Empire, les positions sur les grands enjeux s'étaient fixées et, même si elles restaient susceptibles d'évoluer, les convictions s'étaient forgées. La montée de nouvelles classes sociales, le début du processus de descente de la politique vers les masses, tout ceci créait les conditions d'un débat plus moderne, que le rétablissement de la démocratie pluraliste en 1870-1871 confirma. Les enjeux partisans de l'après-guerre et le mode de scrutin majoritaire retenu en 1876 laisseraientils s'exprimer les subtilités et les sensibilités électorales en gestation? Il est certain en tous les cas, dût-on recourir à des moyens détournés pour l'appréhender, qu'un électorat s'est vite singularisé par un comportement original. Fluctuant entre droite et gauche, on pourrait le croire sans convictions arrêtées. Modérés avant tout, ces électeurs avaient fait du refus des extrêmes le fondement de leur identité politique.

\section{Les modérés, un électorat capital dans la lutte politique}

Le résultat brut d'une consultation ne saurait révéler par lui-même l'existence de l'électorat modéré. La logique majoritaire du scrutin conduit en effet à des schématisations caricaturales : la diversité des sensibilités tend à disparaître derrière les nécessités du combat bipolaire. La comparaison attentive de scrutins successifs, seule, permettra d'appréhender son existence. Toutefois, les jeux d'appareils laissent deviner que son poids est vite apparu déterminant.

La constitution dans le Finistère, en février 1871, d'une troisième liste, dite de conciliation, concurrente donc des listes royaliste et républicaine, a-t-elle été une manifestation véritable d'un tempérament politique spécifique? Pouvait-on véritablement parler d'une " troisième " liste en tant que telle? Les promoteurs de cette " conciliation " avaient en effet simplement substitué à quatre personnalités de la liste des droites deux noms empruntés à la liste républicaine (Lebreton et Rousseau) et deux autres noms (l'ancien député de la Monarchie constitutionnelle Le Bastard de Kerguiffinec et le républicain Auguste Morvan). Pour treize candidats par liste, la liste de conci- 
liation reprenait les deux tiers de la liste des droites. Pour les républicains, il ne s'agissait là que d'une manœuvre pour diviser leurs forces. Il serait en effet plus facile à des électeurs de droite de replacer in extremis les personnalités exclues qu'à des électeurs de gauche de panacher leur bulletin. Les résultats montrent que cela se produisit. Dans le canton de Quimper par exemple, il est aisé de calculer le nombre de voix perdues par la liste républicaine. Les candidats figurant simultanément sur la liste de conciliation et sur la liste républicaine (Rousseau et Lebreton) obtenaient 558 suffrages en plus de leurs collègues de gauche. Ces voix provenaient nécessairement de suffrages s'étant portés sur la liste de conciliation. De fait, c'était là le score moyen de Morvan et de Kerguiffinec. Dans le canton de Quimper, face à 1305 conservateurs et à 971 républicains, 558 électeurs avaient opté pour la conciliation.

Doit-on retenir l'hypothèse d'un " coup monté " des conservateurs? S'il est évident que la liste républicaine a été la principale victime de ces défections, la constitution d'une liste de compromis révèle qu'une partie des élites et des électeurs ne se satisfaisait déjà pas des termes du débat que les états-majors départementaux entendaient imposer. En effet, plusieurs personnalités conservatrices exclues de la liste de conciliation étaient très " marquées " politiquement, comme le vicomte Paul de Forsanz ou le comte Henri de Legge.

Dès les débuts du nouveau régime, la nécessité s'est fait sentir pour les états-majors de courtiser l'électorat modéré et d'éviter, par des choix trop marqués, d'effrayer les électeurs. Dans le Finistère, les élections partielles du 2 juillet 1871 le montrent bien. Par suite du décès ou du départ de quatre députés conservateurs, de nouvelles législatives furent organisées. À droite, deux tendances se dégageaient. Face aux " durs " derrière le journal L'Impartial du Finistère, une ligne plus mesurée s'affirma. Plus proche du terrain, sentant mieux les évolutions de l'esprit public, cette seconde sensibilité n'entendait pas se laisser enchaîner aux monarchistes extrémistes, dont les élections municipales du printemps avaient signé le recul. La tendance modérée l'emporta donc au sein des comités qui supervisèrent la désignation des candidats. Annonçant la constitution de la liste, le journal ultra-légitimiste L'Impartial du Finistère ne pouvait s'empêcher le 28 juin de s'interroger : n'aurait-il pas mieux valu s'en remettre aux seuls notables sans écouter Dieu sait quelles influences? Avec 25000 voix supplémentaires par rapport à février 1871, les quatre républicains passèrent tous. Deux ans plus tard, en 1873, le républicain Gustave Swiney, accroissant encore son avance, s'imposait à son tour lors d'une partielle face à Pierre Le Guen. Ce dernier avait été préféré à Raoul de Raismes, conseiller général d'Arzano et candidat de L'Impartial, jugé peu apte à attirer l'électorat rural modéré (en raison de sa particule).

\section{Un électorat fluctuant?}

L'instabilité entre droite et gauche caractérise en apparence le comportement politique d'une frange de l'électorat qui, au demeurant, n'hési- 
tait pas à se réfugier dans l'abstention pour n'avoir pas à faire un choix contraire à ses convictions. Ce faisant, cet électorat adoptait une attitude très moderne. On peut ainsi affirmer qu'il manifestait une certaine fidélité politique, en faisant prévaloir sur toute autre considération la recherche de la mesure, de l'équilibre et de la solution la moins éloignée de cet idéal de modération.

- L'abstention, un choix par défaut

Les législatives de février 1871 furent marquées par une abstention très élevée. En même temps que par l'absence de nombreux électeurs retenus par leurs obligations militaires, il semble qu'il faille l'expliquer par un choix déterminé et conscient d'une partie de l'électorat, soucieuse de rejeter tout autant le caractère belliciste de la liste gambettiste que le royalisme affiché par les candidats conservateurs.

Tableau 1 - Comparaison des résultats des élections de février 1871, juillet 1871 et décembre 1873 dans le canton de Saint-Renan (\% des inscrits et nombre de voix) ${ }^{1}$

\begin{tabular}{|cc|c|c|c|c|}
\hline \multicolumn{2}{|c|}{1871 (février) } & \multicolumn{2}{c|}{1871 (juillet) } & \multicolumn{2}{c|}{1873 (décembre) } \\
\hline Abstention & $43,7 \% / 1546$ & Abstention & $38,2 \% / 1388$ & Abstention & $23,7 \% / 829$ \\
\hline $\begin{array}{c}\text { Liste } \\
\text { conservatrice }\end{array}$ & $50,6 \% / 1793$ & $\begin{array}{c}\text { Liste } \\
\text { conservatrice }\end{array}$ & $28,3 \% / 1031$ & $\begin{array}{c}\text { Pierre } \\
\text { Le Guen }\end{array}$ & $50,1 \% / 1751$ \\
\hline $\begin{array}{c}\text { Liste } \\
\text { républicaine }\end{array}$ & $5,7 \% / 201$ & $\begin{array}{c}\text { Liste } \\
\text { républicaine }\end{array}$ & $33,0 \% / 1201$ & $\begin{array}{c}\text { Gustave } \\
\text { Swiney }\end{array}$ & $26,1 \% / 913$ \\
\hline
\end{tabular}

Tableau 2 - Comparaison des résultats des élections de 1871 et 1873 dans le canton de Ploudalmézeau (\% des inscrits et nombre de voix)

\begin{tabular}{|c|c|c|l|c|c|}
\hline \multicolumn{2}{|c|}{1871 (février) } & \multicolumn{2}{c|}{1871 (juillet) } & \multicolumn{2}{c|}{1873 (décembre) } \\
\hline Abstention & $43,1 \% / 1625$ & Abstention & $38,2 \% / 1576$ & Abstention & $27,9 \% / 1134$ \\
\hline $\begin{array}{c}\text { Liste } \\
\text { conservatrice }\end{array}$ & $54,0 \% / 2037$ & $\begin{array}{c}\text { Liste } \\
\text { conservatrice }\end{array}$ & $28,2 \% / 1162$ & $\begin{array}{c}\text { Pierre } \\
\text { Le Guen }\end{array}$ & $41,9 \% / 1700$ \\
\hline $\begin{array}{c}\text { Liste } \\
\text { républicaine }\end{array}$ & $2,1 \% / 81$ & $\begin{array}{c}\text { Liste } \\
\text { républicaine }\end{array}$ & $33,9 \% / 1397$ & $\begin{array}{c}\text { Gustave } \\
\text { Swiney }\end{array}$ & $31,0 \% / 1259$ \\
\hline
\end{tabular}

Dans l'indécision qui a régné au lendemain de la défaite face à la Prusse, sommés de choisir entre une liste monarchiste et une autre belliciste, nombre d'électeurs du Finistère se réfugièrent dans l'abstention en février 1871. Quatre mois plus tard, ils votaient à gauche, rassurés tout

1. PAUBERT, Laurent, Formation de l'opinion publique, politisation et structuration des identités politiques en Bretagne au XIX siècle. L'exemple du Finistère et du Morbihan de 1848 à 1914, Thèse de doctorat d'histoire soutenue à Brest en janvier 2003. (Tous les tableaux contenus dans cet article sont issus de ce travail.) 
autant par la fermeté des républicains face à la Commune que par les personnalités présentées. En 1873, une partie, sans doute de sensibilité conservatrice mais non monarchistes, repassait à droite.

Tableau 3 - Comparaison des résultats des élections législatives générales de 1871 et des partielles de 1873 dans le canton de Gourin (\% des inscrits et nombre de voix)

\begin{tabular}{|cc|cc|}
\hline \multicolumn{2}{|c|}{1871} & \multicolumn{2}{|c|}{1873} \\
\hline Abstention & $64,2 \% / 2016$ & Abstention & $39,4 \% / 1162$ \\
Score des conservateurs & $34,2 \% / 1076$ & Score de Charles du Bodan & $35,6 \% / 1050$ \\
Score des républicains & $1,6 \% / 49$ & Score du républ. Beauvais & $24,9 \% / 734$ \\
\hline
\end{tabular}

Tableau 4 - Comparaison des résultats des élections de 1871 et de 1873 dans le canton de Locminé (\% des inscrits et nombre de voix)

\begin{tabular}{|lc|cc|}
\hline \multicolumn{2}{|c|}{1871} & \multicolumn{2}{|c|}{1873} \\
\hline \multicolumn{1}{|c|}{ Abstention } & $44,9 \% / 1403$ & Abstention & $25,3 \% / 785$ \\
Score des conservateurs & $49,6 \% / 1552$ & Score de Charles du Bodan & $49,1 \% / 1524$ \\
Score des républicains & $5,5 \% / 173$ & Score du républ. Beauvais & $25,6 \% / 793$ \\
\hline
\end{tabular}

Tableau 5 - Comparaison des résultats des élections de 1871 et de 1873 dans le canton d'Allaire (\% des inscrits et nombre de voix)

\begin{tabular}{|cc|cc|}
\hline \multicolumn{2}{|c|}{1871} & \multicolumn{2}{|c|}{1873} \\
\hline Abstention & $47,9 \% / 1598$ & Abstention & $32,3 \% / 1072$ \\
Score des conservateurs & $47,4 \% / 1584$ & Score de Charles du Bodan & $47,9 \% / 1590$ \\
Score des républicains & $4,7 \% / 157$ & Score du républ. Beauvais & $19,7 \% / 655$ \\
\hline
\end{tabular}

Tableau 6 - Comparaison des résultats des élections de 1871 et 1873 dans le canton de Mauron (\% des inscrits et nombre de voix)

\begin{tabular}{|cc|cc|}
\hline \multicolumn{2}{|c|}{1871} & \multicolumn{2}{|c|}{1873} \\
\hline Abstention & $41,0 \% / 1075$ & Abstention & $24,2 \% / 542$ \\
Score des conservateurs & $58,9 \% / 1543$ & Score de Charles du Bodan & $47,7 \% / 1069$ \\
Score des républicains & - & Score du républ. Beauvais & $28,0 \% / 626$ \\
\hline
\end{tabular}

Comme dans le Finistère, on observe que de nombreux électeurs morbihannais se sont détournés de la liste monarchiste en février 1871. Ils ont préféré se réfugier dans l'abstention plutôt que de voter pour la liste gambettiste. Comme dans le Finistère, la gauche a progressé notablement au fil des années, parvenant même parfois à « mordre " sur les adversaires.

Il peut sembler difficile de voir dans cet électorat fluctuant l'esquisse d'une personnalité politique modérée, à une époque où les identités politiques ne sont pas encore très précises et où l'autonomie électorale n'est 
que relative. Pourtant, la persistance sur la durée de ce comportement politique original, ignorant les cloisonnements et les frontières trop affirmées, est une donnée marquante de la vie politique bretonne.

\section{- Apparentes oscillations politiques de l'électorat modéré}

Les législatives de 1876 et 1877 ont confirmé le rôle essentiel de cet électorat modéré. Consciente de ce poids, la gauche avait investi deux républicains catholiques, Joseph de Gasté à Brest et Joseph Nédellec à Carhaix, ainsi qu'un républicain conservateur à Pont-l'Abbé, Georges Arnoult. La comparaison des résultats des élections de 1876 et de 1877 est extrêmement enrichissante. Elle seule permet de révéler l'existence de cet électorat. On comprend en tous les cas que les états-majors aient souhaité séduire ces électeurs : il était essentiel de les gagner à sa cause car d'eux dépendait le sort d'une élection. Observons leur attitude dans deux circonscriptions, Châteaulin et Quimperlé.

Dans la circonscription de Châteaulin, le candidat des droites en 1877 fut l'ultraroyaliste comte Henri de Legge. L'année précédente, il avait été vaincu dans la circonscription voisine de Carhaix par le républicain catholique Joseph Nédellec. Échaudé par cet échec, il avait préféré cette fois changer de circonscription. En 1876 et en 1877, le candidat de gauche était le républicain Théophile de Pompéry. Cette similitude autorise les comparaisons.

Tableau 7 - Comparaison des résultats aux législatives dans le canton de Châteaulin entre 1871 et 1877

\begin{tabular}{|c|c|c|c|c|}
\hline Scrutins & Inscrits & Abstentions & $\begin{array}{c}\text { Suffrages } \\
\text { de droite }\end{array}$ & $\begin{array}{c}\text { Suffrages } \\
\text { de gauche }\end{array}$ \\
\hline Février 1871 & 4604 & 3115 & 709 & 880 \\
Juillet 1871 & 4669 & 2018 & 772 & 1862 \\
1873 & 4574 & 1423 & 1205 & 1948 \\
1876 & 4486 & 1160 & 2101 & 1205 \\
1877 & 4609 & 993 & 1249 & 2392 \\
\hline
\end{tabular}

On constate que, dans le canton de Châteaulin, la droite a nettement accru ses scores jusqu'en 1876, année où le conservateur modéré Chauvel se présenta. L'arrivée de Henri de Legge en 1877 provoqua l'érosion de cet électorat, ramenant la droite à son score de 1873. À l'évidence, une partie de la droite, conservatrice certes, mais hostile aux idées trop exaltées, à été désarçonné par le parachutage d'un royaliste aux convictions ultra bien connues. Dans le canton de Crozon également, la personnalité du comte de Legge a conduit un certain nombre d'électeurs Chauvel de 1876 à faire le choix de Pompéry l'année suivante. 
Tableau 8 - Comparaison des résultats aux législatives dans le canton de Crozon entre 1871 et 1877

\begin{tabular}{|c|c|c|c|c|}
\hline Scrutins & Inscrits & Abstentions & $\begin{array}{c}\text { Suffrages } \\
\text { de droite }\end{array}$ & $\begin{array}{c}\text { Suffrages } \\
\text { de gauche }\end{array}$ \\
\hline Février 1871 & 4130 & 1947 & 1511 & 672 \\
Juillet 1871 & 4185 & 1796 & 546 & 1814 \\
1873 & 4097 & 1333 & 282 & 2469 \\
1876 & 4262 & 1082 & 901 & 2186 \\
1877 & 4261 & 930 & 723 & 2598 \\
\hline
\end{tabular}

La circonscription de Quimperlé permet les mêmes constatations. En 1876, l'ancien député impérialiste Louis du Couëdic, un légitimiste rallié à Napoléon III, ultramontain et clérical ardent, parvint à imposer sa candidature aux chefs de la droite. Il fut vaincu par un républicain modéré, catholique lui aussi, Corentin Guyho. L'année suivante, conscients du rejet dont avait souffert leur candidat l'année précédente, les conservateurs investirent un orléaniste, Lorois. Et de fait, celui-ci parvint à mordre sur l'électorat de son rival et emporta la victoire. Il était en effet parvenu à récupérer une partie des modérés. Une partie de ces gains étaient également dues à des manœuvres éhontées et à des pressions excessives : son élection fut invalidée. En 1878, Corentin Guyho reconquit une partie des positions perdues au centre de l'échiquier politique. Cela fut suffisant pour lui assurer une nouvelle fois le siège.

\section{Tableau 9 - Comparaison des résultats des législatives dans le canton de Pont-Aven entre 1871 et 1877}

\begin{tabular}{|c|c|c|c|c|}
\hline Scrutins & Inscrits & Abstentions & $\begin{array}{c}\text { Suffrages } \\
\text { de droite }\end{array}$ & $\begin{array}{c}\text { Suffrages } \\
\text { de gauche }\end{array}$ \\
\hline Juillet 1871 & 2941 & 1365 & 955 & 614 \\
1873 & 3097 & 839 & 896 & 1361 \\
1876 & 3109 & 846 & 866 & 1427 \\
1877 & 3288 & 581 & 1433 & 1331 \\
1878 & 3370 & 784 & 1055 & 1507 \\
\hline
\end{tabular}

Savoir se gagner les suffrages des électeurs modérés et les conserver représentait bien souvent un atout pour la victoire finale. Par essence ennemis des solutions radicales, ils inclinaient soit à droite, soit à gauche. On peut les qualifier d'électorat central. L'évolution des données du débat politique, avec l'éloignement de la perspective d'une restauration puis avec l'appel du pape au ralliement à la République, avec la montée des questions sociales, finirent par donner à cet électorat un poids tout à fait crucial. Un corps électoral plus réduit et un scrutin à deux degrés lors des élections sénatoriales, loin de démentir le rôle de ce "centre " politique, en facilitent au contraire la compréhension. 


\section{Les sénatoriales, moment par excellence de la conjonction des centres au début du vingtième siècle}

Les années 1900-1914, après plus d'un demi-siècle de fonctionnement du suffrage universel, permettent d'observer les attitudes d'électeurs confrontés à des choix majeurs. L'appel au Ralliement du pape Léon XIII en 1892, l'Affaire Dreyfus et la montée du nationalisme, l'acuité de la question sociale et de la querelle religieuse, sont autant d'éléments qui déterminèrent une profonde mutation des frontières politiques. À propos du début du Xx ${ }^{\mathrm{e}}$ siècle, Marcel Gauchet considère que " droite et gauche vont s'imposer alors comme les noms par excellence de ces deux France qui s'affrontent passionnément ${ }^{2}$ ». Il constate

" [qu'] à la veille de la guerre de 1914, leur rôle de repère est définitivement consacré. La topographie parlementaire est devenue le moyen primordial par lequel les citoyens se pensent en politique".

André Siegfried, déjà, énonçait que

" deux conceptions de la société, à travers un duel séculaire et passionné se disputent le pays depuis la Révolution. L'une, ayant l'autorité pour base, assied l'équilibre politique sur une hiérarchie d'autorités sociales comme elle établit l'équilibre de l'univers sur la toute-puissance de la divinité. L'autre, basée sur l'égalité et sur la liberté, restitue à chaque citoyen sa part de souveraineté, affirme ses droits plus que ses devoirs, dresse les anciens subordonnés contre leurs anciens maîtres et par là renie la vieille notion de hiérarchie sociale et religieuse ${ }^{3}$ ".

Pierre Milza a pointé les a priori partisans dont souffre le travail du père de la géographie politique. Loin de présenter l'image caricaturale qu'on voudrait lui prêter, le débat politique au début du vingtième siècle montre en effet combien c'était désormais, plus que jamais, au centre que se gagnait une élection.

\section{Dépassement de la bipolarisation et conjonction des centres dans le Finistère}

En 1890, les grands électeurs finistériens portaient pour la première fois un républicain à la Haute-Assemblée. L'étude des élections sénatoriales dans le Finistère après 1893 dément l'image figée d'un face-à-face absolu cléricaux-républicains. Cette situation n'a prévalu en fait que quelques années (1894-1895). Rapidement les rivalités d'ambition à gauche et les querelles sur la stratégie à suivre ont brouillé les clivages, jusqu'à permettre l'élection surprise d'un sénateur de la droite dure (1897). Les conséquences de l'entrée d'un socialiste dans le gouvernement Waldeck-Rousseau annoncèrent bientôt les recompositions à venir. À partir de 1901, dans le climat

2. NoRA, Pierre, Les Lieux de mémoire, Paris, Gallimard, 1992, p. 412-413.

3. Siegrried, André, Tableau politique de la France de l'Ouest sous la Troisième République, Paris, Colin, 1913, xxvIII-536 p. [reprint, Genève, Slatkine, 1980], citation p. 597. 
de passions religieuses propre au début du siècle, se produisit un reclassement politique de grande ampleur, conjonction des centres amorcée dès 1900 dans le Finistère avec l'élection de deux notables, Pichon et Lambert.

\section{- 1900 : année-clé de la recomposition politique}

Les élections sénatoriales du 28 janvier 1900, à la suite du décès des maires de Quimperlé et Châteaulin, manifestèrent les mutations en cours. La pré-campagne fut marquée par l'entrée en lice de deux candidats inattendus, l'ancien député républicain de Brest Louis Pichon et le général Lambert, écarté par le congrès républicain trois ans auparavant. Tous deux étaient des républicains catholiques, irrités par l'entrée d'un socialiste dans le gouvernement Waldeck-Rousseau. La profession de foi de Lambert affirmait :

«Républicain loyal et convaincu, ennemi de toute violence, je ne suis ni bleu, ni blanc, ni rouge mais tricolore. Jamais je ne suivrai les intolérants ou les utopistes."

Le ralliement des conservateurs à cette double candidature entraîna leur facile victoire avec plus de $60 \%$ des voix. Ce résultat consterna les républicains car il révélait qu'une stricte discipline de vote n'avait pas été observée de leur côté. Effrayés par la formation d'un ministère " d'union de la gauche ", dit de Défense républicaine, en 1898, les plus modérés des républicains avaient évolué vers le centre-droit. Ce phénomène était particulièrement net chez les jeunes générations, celles qui n'avaient pas vécu les événements du Seize Mai aussi intensément que leurs aînés et désiraient enterrer des clivages qui leur apparaissaient comme désuets en face du danger socialiste. Ils avaient été déçus par le choix du congrès de Châteaulin, leurs préférences se portant vers un ticket Pichon-Hémon, " qui appartiennent tous deux au parti républicain modéré qui a pour chef Mr Méline " analysait le préfet du Finistère. Ces deux noms étaient tout à fait symboliques du rejet de toute alliance avec l'extrême-gauche. Au moment de la constitution du ministère Waldeck-Rousseau, Louis Hémon avait été désigné comme porte-parole au Parlement des progressistes qui refusèrent d'entrer dans la majorité :

" Nous avons dit et redit pour attirer à la République ceux qui la tenaient en défiance qu'elle serait, contre le collectivisme, la meilleure gardienne, la protection la mieux qualifiée de la société issue de 1789. Que voulez-vous que nous répondions aujourd'hui à tant de braves gens qui exprimeront leur stupéfaction de voir qu'à la porte de la société, parmi ceux qui sont aujourd'hui chargés d'y monter la garde, il y a ceux-là même qui ne rêvaient hier que de l'enfoncer ${ }^{4}$."

4. Le Finistère, 28 janvier 1899, cité dans TrEBAOL C., Louis Hémon (1844-1914), député et sénateur républicain, et la vie politique dans la Cornouaille quimpéroise sous la Troisième République, thèse de $3^{\mathrm{e}}$ cycle, université de Bretagne Occidentale, 1978, 290 p. 
- La bipolarisation démentie :

l'attitude des ralliés et des démocrates chrétiens

Au début de 1901, les ralliés favorisèrent l'élection du candidat de droite Jules de Cuverville en remplacement d'Arsène Lambert. Bientôt pourtant, leur attitude évolua.

Le décès de Joseph Astor cinq mois plus tard entraîna de nouvelles partielles. La préfecture évaluait l'état des forces à 500 républicains environ, 200 démocrates chrétiens et 500 " réactionnaires ". Quelques mois après la parution au Journal Officiel de la loi sur les Associations, on pouvait craindre que la même coalition qu'en mars n'assure une nouvelle fois le succès du candidat des droites. Deux hommes se retrouvèrent en lice : le maire républicain modéré de Quimper Adolphe Porquier et le maire de Daoulas, Danguy des Déserts. Ce dernier disposait de solides soutiens chez les royalistes. Sa profession de foi explicitement favorable au régime lui assurait en outre l'appui des ralliés de la tendance d'Albert de Mun. Mais le soutien affiché des monarchistes discréditait Danguy des Déserts aux yeux des démocrates-chrétiens tendance abbé Gayraud. En face, Adolphe Porquier, qui se prononçait dans sa profession de foi pour une application très libérale de la législation laïque, emportait leurs sympathies. Par la voix de leur journal L'Étoile de la Mer, les démocrates tendance abbé Gayraud appelèrent à voter pour le maire de Quimper. Fort de ce soutien, Porquier remporta le scrutin du 11 novembre 1901. La droite n'eut de cesse alors de dénoncer cette collusion qui semblait inimaginable. Ce faisant, le centre démocrate-chrétien avait manifesté sa parfaite indépendance et démontrait qu'il entendait transcender une bipolarité jugée artificielle pour faire prévaloir la ligne la plus mesurée.

L'année suivante, le préfet confirmait, en prévision des sénatoriales générales de 1903, l'importance des démocrates-chrétiens, au centre de l'échiquier politique et écrivait que

" depuis plusieurs années le corps électoral du Finistère comprend trois partis : le parti réactionnaire, contenant les débris de l'ancien parti royaliste, le parti catholique rallié du Léon et les républicains de nuances diverses. Ce sont les ralliés catholiques qui feront le départage et entraîneront l'élection ${ }^{5}$ ".

Les législatives de 1902 avaient ouvert les portes du pouvoir au parti radical. La politique antireligieuse fut intensifiée. Le sous-préfet de Brest écrivait le 25 août 1902 que

" le corps électoral se compose de trois éléments : les réactionnaires irréductibles, parmi lesquels je compte les royalistes, les catholiques ralliés et les républicains de toutes nuances. Les seconds sont de beaucoup les plus nombreux et c'est d'eux uniquement que dépend le succès. Ils se portent alternativement à droite ou à gauche suivant leurs intérêts du moment. Il n'est pas douteux que dans la situation actuelle, ils se portent du côté des réactionnaires".

5. Arch. dép. du Finistère, 3 M 339, rapport du préfet, 16 septembre 1902. 
Un exemple précis nous est fourni dans l'arrondissement de Quimperlé. Dans la commune de Tourch, le conseil municipal issu des élections de 1900 se composait de quatre républicains, deux ralliés et cinq réactionnaires. Les ralliés avaient contribué à porter à la tête de la municipalité le républicain Postic. Or, à l'approche du scrutin sénatorial, ils firent désigner deux grands électeurs de droite. Inquiet de cette évolution, particulièrement accentuée dans l'arrondissement de Brest, le préfet prodiguait ses conseils :

"Les ralliés du Léon, qui avaient avant les décrets du ler août une tendance à se rapprocher du vieux parti républicain sur le terrain de la pensée et de l'enseignement, vont [désormais] se porter sur les candidats de quelque nuance politique que ce soit qui leur offriront le plus de garanties au point de vue catholique et ce sont ceux-là qui seront élus. La liste républicaine ne pourra être qu'une liste très modérée et dont tous les noms appartiendront sous peine d'un échec certain à des défenseurs plus ou moins avoués des congrégations."

Une fois encore, les ralliés détenaient entre leurs mains le sort de l'élection. Ils favorisèrent la réélection de tous les sénateurs sortants : Chamaillard, Cuverville, Pichon pour la droite, Porquier, Delobeau pour la gauche. Une logique de conjonction des centres se mettait peu à peu en place.

- Le triomphe des modérés : l'élection se gagne au centre

En novembre 1903, les mesures combistes avaient davantage encore qu'en janvier brouillé les clivages. Le décès d'Adolphe Porquier, après plusieurs mois d'agonie, entraîna la convocation de nouvelles élections. "L'application de la loi de 1901 dans ce milieu foncièrement catholique et plus encore les restrictions apportées à l'usage de la langue bretonne ont constitué dans le Finistère un bloc antiministériel qui comprend la grande majorité des électeurs " observait le préfet en août 1903. Les libéraux, par la voix de leur organe, L'Action libérale de Quimper, firent savoir qu'ils plaçaient leurs espoirs dans la candidature de l'ancien maire progressiste de Châteaulin, Armand Gassis. Il avait été récemment révoqué pour s'être opposé à l'application des expulsions des congrégations enseignantes. Or, refusant un tel parrainage, Gassis entendit d'abord faire confirmer sa candidature par le congrès républicain. La majorité de celui-ci, nettement plus à gauche, donna son investiture au conseiller général radical de Rosporden Herland. Refusant de s'incliner, Armand Gassis maintint sa candidature, appuyé par l'ensemble de la droite modérée. L'alliance des progressistes conservateurs et de la droite aboutit à son succès avec 70 \% des grands électeurs. Le centre triomphait.

Après le décès de Henri de Chamaillard en 1908, le président du Conseil général, James de Kerjegu, souhaita briguer sa succession. Il s'agissait d'une personnalité de gauche très modérée, ancien monarchiste devenu républicain par refus du boulangisme. Il s'était nettement situé dans l'opposition à 
la politique combiste. Il était soutenu par le grand journal progressiste du département, La Dépêche de Brest. Ses votes au Parlement, rejoignant souvent ceux de la droite, lui garantissaient les suffrages des grands électeurs conservateurs. La majorité qui avait fait l'élection de Gassis lui semblait promise. Pour cette raison même, il était très contesté à gauche. Le congrès d'investiture tenu à Châteaulin à la fin mai rejeta sa candidature pour lui préférer celle du conseiller général de Pont-Croix, Maurice Fenoux. L'affrontement longtemps contenu entre républicains modérés et anticléricaux éclatait. Le républicain modéré de Landerneau Léopold Maissin décida de se présenter pour ne pas laisser libre l'espace du centre-gauche. Le conseiller municipal libéral de Loctudy Henri de Carfort se lança pour la droite. Ils étaient aux yeux de beaucoup incapables de rassembler l'ensemble de l'électorat modéré et de faire barrage aux radicaux. C'est pourquoi le président départemental des libéraux Jules Fortin se lança en personne dans la course. Ce dernier ne l'emporta qu'avec 50,26 \% des voix. L'éloignement des querelles liées à la question religieuse avait encouragé une partie des républicains modérés, des progressistes, à voter plus à gauche.

Mais, peu à peu, une majorité des progressistes passèrent du centregauche au centre-droit. L'élection du président du Conseil général, après le décès de James de Kerjégu, manifesta ce renversement des alliances. L'élection opposa en avril 1909 le député de Carhaix Dubuisson, qui s'était rapproché des radicaux, et le sénateur-maire progressiste de Brest Delobeau. Appuyé par une majorité de centre-droit, ce dernier fut élu. L'année 1909 marque ainsi une nouvelle étape dans l'histoire politique du Finistère.

Le renouvellement de la représentation sénatoriale en 1912 amena la constitution d'une liste unique progressistes-libéraux. Elle comprenait Fortin et Pichon pour les libéraux, Gassis et Delobeau pour les progressistes et le lieutenant-colonel Roudière pour les nationalistes. Derrière Le Progrès du Finistère, les éléments les plus conservateurs parrainèrent la candidature parallèle de Jules de Cuverville. La situation à gauche s'était aussi recomposée. Une fraction des progressistes, derrière Louis Hémon, avait refusé de suivre Delobeau et ses partisans. Profondément réservé sur une alliance avec les radicaux, eux-mêmes tentés par une ouverture vers les socialistes, Hémon accepta malgré tout de figurer sur une liste unique de la gauche. Cette liste "blocarde " comme disait le Courrier du Finistère se composa finalement de trois républicains de gauche, Louis Hémon, Maurice Fenoux et le conseiller général de Morlaix Léon Briens, et de deux radicaux, Louis Dubuisson et Alain Piton, conseiller général de Brest. Trois candidats l'emportèrent dès le premier tour : Pichon, Delobeau et Fortin. Hémon l'emporta au second tour, Fenoux au troisième contre de Cuverville. Par ses votes, rejetant tout extrême, la majorité modérée du collège sénatorial avait donné une juste représentation du Finistère politique en cette avant-guerre de 1914 : trois élus de centre-droit, deux élus de centre-gauche.

En juillet 1912, après le décès de Delobeau, la majorité de centre-droit élit sénateur le libéral Émile Villiers, député de Landerneau. La victoire d'un 
candidat très conservateur traduisait une certaine droitisation du département. La faible majorité qu'il obtint indiquait cependant qu'il suscitait un certain nombre de réticences parmi la droite modérée. L'élection sénatoriale du 31 mai 1914, dans un contexte de lutte scolaire réactivée par les projets de monopole agités par les radicaux, réactiva les clivages traditionnels. Le candidat des droites, le maire libéral de Douarnenez Louis de Pénanros, réédita quasiment le score de Villiers : 50,91 \% des voix.

\section{Le comportement électoral des modérés au début du $\mathrm{xx}^{\mathrm{e}}$ siècle}

Les élections sénatoriales ont montré l'importance des électeurs modérés, dont l'appoint était indispensable pour former une majorité. Ils se sont également trouvés en mesure de faire la différence lors de nombreuses élections législatives. L'essentiel, électoralement parlant, se passait au centre de l'échiquier. Les états-majors en étaient conscients, qui adaptaient leurs discours pour séduire ces électeurs modérés, aux suffrages volatiles, dont le choix ferait en définitive le succès ou la défaite.

\section{Villes et campagnes républicaines du Morbihan. Deux milieux, deux sensibilités, un même défi : séduire l'électeur}

Compte-tenu du poids du monde rural et du découpage des circonscriptions, le seul espoir de l'emporter pour les républicains résidait dans la possibilité de gagner des voix dans les campagnes.

En 1893 dans la circonscription d'Auray-Hennebont, le républicain modéré Eugène Le Coupanec, fils de paysans aisés, parvint à s'imposer contre le sortant Gustave de Lamarzelle en obtenant les suffrages de l'électorat rallié. Sa profession de foi faisait vibrer la corde populaire :

" Je n'ai à vous présenter dans ma famille ni comte, ni baron, ni marquis mais je pourrais vous offrir en grand nombre des paysans, des pêcheurs, des ouvriers, des commerçants et j'en suis fier."

Sa personnalité séduisait l'électorat modéré. Au Parlement, il vota contre l'impôt sur le revenu, rejoignant le sénateur Lamarzelle, son ancien adversaire. Maire de Ploemeur, Le Coupanec fit autoriser en 1903 par son conseil l'installation de la congrégation des filles de la Sagesse car, disaitil, la suppression des écoles congréganistes serait la ruine de tous les budgets communaux.

Un congrès républicain à Auray désigna en 1898 pour lui succéder un cultivateur de Landévant Joseph Jacob. C'était là un choix habile puisque, si les ancrages à gauche dans les villes et sur le littoral paraissaient solides, selon le sous-préfet, " c'est la partie cultivatrice de la région qu'il s'agit de ramener [à la République] ${ }^{6}$ ". La modération de Jacob lui gagna en effet de nombreux soutiens. Ainsi à Languidic, " grosse commune jusqu'à présent

6. Arch. dép. du Morbihan, M 4715, rapport du sous-préfet de Lorient, 3 février 1898. 
entièrement acquise aux réactionnaires, $\mathrm{Mr}$ Jacob compte [désormais] de nombreuses sympathies qui se traduisent par le fait que son journal $L a$ République du Morbihan s'y vend par centaines de numéros ${ }^{7}$ ". Son adversaire royaliste, de Goulaine, était donné vainqueur au début de la campagne. En parvenant à séduire l'électorat central, Jacob l'emporta finalement.

La séduction exercée sur l'électorat par des républicains modérés était évidente. La circonscription de Vannes en offre aussi l'illustration la même année. Le légitimiste Charles du Bodan, président du comité catholique, était son député depuis 1871. " Une tentative a été faite en 1893 mais, contre Mr du Bodan, elle ne pouvait réussir. On votait pour lui surtout par sympathie personnelle " écrivait le préfet. Son décès à la fin de l'année 1897 allait permettre à l'électorat républicain de s'exprimer. La droite désigna pour briguer le siège vacant le président de la Société Polymathique du Morbihan, Régis de L'Estourbeillon. En 1893, Charles du Bodan avait gagné avec 5500 voix de plus que son compétiteur, le commandant Taillandier. En janvier 1898, Régis de L'Estourbeillon remporta l'élection partielle avec moins de 2500 voix d'avance.

Comment expliquer ces mouvements de l'opinion? En fait, le nombre des conservateurs avait toujours été inférieur dans la circonscription aux suffrages de du Bodan, qui bénéficiait d'un vote personnel plus que politique. En 1898, les républicains eurent l'intelligence de présenter un candidat capable de rallier l'électorat du centre. Les débats furent âpres au sein des instances dirigeantes du parti. On en connaît la teneur par la presse. Le 5 janvier 1898, le journal départemental d'action républicaine Le Progrès du Morbihan relatait la tenue d'un congrès des divers comités ruraux de la circonscription. Ceux-ci imposèrent aux Vannetais leur candidat, l'ancien conseiller général de Vannes-Ouest, Achille Martine. C'était un choix habile. Sa fiche administrative notait qu'«il n'a[vait] jamais manifesté de nuance spéciale mais il a[vait] dans plusieurs réunions publiques déclaré qu'il était acquis à la politique gouvernementale [Méline] et il sa[va]it que la circonscription de Vannes ne peut venir à la République qu'avec des promesses de grande modération ". Le calcul se révéla payant. De nombreux républicains ruraux, abstentionnistes jusque-là, contribuèrent à son succès. Les élections générales du 8 mai élargirent encore cette victoire : Régis de l'Estourbeillon ne distançait plus le candidat républicain que de 1308 voix. L'électorat de centre-gauche s'était jusqu'à présent abstenu ou avait voté par sympathie ou obéissance pour Charles du Bodan. Son réveil fragilisait indéniablement la domination de la droite vannetaise.

\section{Querelles de sensibilités dans la droite finistérienne : la question des sillonistes}

L'élection d'Albert de Mun en 1894 et celle d'Hippolyte Gayraud en 1897 avaient témoigné de l'implantation du courant démocrate-chrétien dans le

7. Arch. dép. du Morbihan, M 4715, rapport du sous-préfet de Lorient, 16 avril 1898. 
Léon. Le mouvement de Marc Sangnier, le Sillon, avait tissé de puissants réseaux dans le Finistère. Sa condamnation par le pape Pie X dans une lettre aux évêques français en août 1910 l'amena à se saborder. "Alors que de nombreux sillonistes se rapprochent de l'ACJF, mouvement plus vaste mais aussi plus prudent qui reçoit l'approbation de bien des membres de la hiérarchie catholique, un groupe assez restreint de militants attachés à l'ancien Sillon optent pour une voix différente. Libérés de toute autorité, ils décident d'entreprendre, sous leur propre autorité, une action politique afin de défendre et promouvoir les idées républicaines et démocratiques auxquelles ils ont toujours adhéré ${ }^{8}$." Cette volonté se concrétisa dans la fondation en 1911 de la Fédération des Républicains Démocrates du Finistère (FRD). Son leader départemental était le conseiller général de Plouescat Pierre Trémintin. Ses lieutenants principaux s'appelaient Victor Balanant, ancien militant de la CGT, Paul Simon, Henri Le Goasguen, Adolphe Le Goaziou, Jacques Fonlupt ou Jean Jadé. Tous furent appelés dans les années suivantes à briguer des mandats législatifs. La FRD se réclamait d'un humanisme d'inspiration chrétienne. Elle proclamait son attachement à la République, sa volonté de justice sociale et de réforme politique. Ses membres étaient tous d'ardents catholiques. Alors que les libéraux, héritiers du Ralliement, tendaient à se rapprocher de la droite, les sillonistes occupèrent l'espace laissé vacant au centre de l'échiquier politique.

- L'élection de Paul Simon en 1912 : sensibilité démocrate contre sensibilité libérale

L'implantation et les réseaux démocrates leur permirent rapidement de jouer un rôle politique majeur. En 1913, Paul Simon se présenta dans la circonscription de Landerneau où se disputait la succession d'Emile Villiers, élu sénateur l'année précédente. Trois autres candidats revendiquaient le siège : un socialiste, Jean-François Le Gall, un radical, Sauveur Salahun, un libéral de Landerneau, Gaston de L'Hôpital. Il était parrainé par Villiers et par l'évêque de Quimper. Il avait pour lui l'aristocratie et le vieux clergé. Il s'affirmait républicain mais ses déclarations très cléricales lui avaient aliéné l'électorat de centre-gauche. L'encadrement très aristocratique de sa campagne mécontentait les électeurs de centre-droit. C'est dans ces conditions que se produisit la candidature de Paul Simon. Il était soutenu par le jeune clergé.

La lutte entre le républicain libéral et le républicain démocrate tourna à l'avantage du second. À quelques jours du premier tour, le préfet écrivait que

" ces divisions du parti clérical ne sont peut-être pas nouvelles mais c'est la première fois qu'elles se manifestent ici au grand jour. Elles sont graves de conséquences pour les réactionnaires et déjà la lutte a pris un carac-

8. Floch, J.-C., Les Républicains démocrates du Finistère (1911-1940), Mémoire de mâ̂trise d'histoire, Université de Bretagne Occidentale, 1990, 229 p. 
tère de violence et d'âpreté qui rendra tout rapprochement difficile, incertain, et pourra dans l'avenir, si elle se généralise profiter au parti républicain".

Un rapport du préfet du Finistère rendait à nouveau compte entre les deux tours de l'impact probable de la victoire d'un républicain-démocrate à Landerneau et de "l'intérêt considérable qui s'attachait à cette élection dont les conséquences pourront orienter vers une nouvelle direction la politique de ce département ". Il apparaissait clairement au fonctionnaire que la sensibilité des sillonistes s'accordait parfaitement aux attentes des populations.

« Le sillonisme répond à l'état d'esprit des Bretons. Et c'est là son danger. Il représente exactement l'expression, la formule de la pensée de cette race à la fois croyante et par tempérament comme par reconnaissance attachée au gouvernement républicain. L'élection de Mr Simon donnerait à ce parti encore en formation un essor considérable. Il pourrait devenir menaçant non seulement pour les catholiques traditionalistes mais aussi pour les députés républicains."

Le sillonisme apparaissait comme l'incarnation parfaite du " centre ", le mieux à même d'incarner les attentes de l'électorat central, à la césure de la droite et de la gauche, amalgamant le centre-gauche républicain catholique et le centre-droit démocrate-chrétien, issu du ralliement mais délesté de ses éléments les plus conservateurs ou libéraux.

- Électorat de gauche, électorat de droite, électorat silloniste :

la question des reports

Le préfet du Finistère avait compris les dangers que représentait la démocratie chrétienne, à la charnière des territoires symboliques traditionnels de la droite et de la gauche ${ }^{9}$. L'étude du comportement politique des électorats renseignera à la fois sur les affinités des uns et des autres et sur leur perception de leurs identités politiques respectives.

En 1913, dans la circonscription de Landerneau, les démocrates-chrétiens concoururent donc pour la première fois aux élections législatives en la personne de Paul Simon. En 1910, on avait compté 5980 inscrits. En 1913, 5994. On comptait 400 votants supplémentaires au premier tour en 1913, 550 au second tour.

\section{Tableau 10 - Comparaison des résultats des élections législatives de 1910 et 1913 dans le canton de Daoulas (nombre de suffrages et \% des inscrits)}

\begin{tabular}{|c|c|c|}
\hline Candidats 1910 & Républicain libéral & Socialiste \\
\hline Scores & $2541(42,49 \%)$ & $690(11,5 \%)$ \\
\hline
\end{tabular}

9. Arch. dép. du Finistère, 3M 311, rapport du préfet du Finistère, 9 avril 1913. 


\begin{tabular}{|c|c|c|c|c|}
\hline $\begin{array}{c}\text { Candidats } 1913 \\
\left(1^{\mathrm{er}} \text { tour }\right)\end{array}$ & $\begin{array}{c}\text { Républicain } \\
\text { libéral }\end{array}$ & $\begin{array}{c}\text { Républicain } \\
\text { démocrate }\end{array}$ & $\begin{array}{c}\text { Radical- } \\
\text { socialiste }\end{array}$ & Socialiste \\
\hline Scores & $1182(19,8 \%)$ & $1642(27,4 \%)$ & $683(11,4 \%)$ & $187(3,1 \%)$ \\
\hline
\end{tabular}

\begin{tabular}{|c|c|c|c|}
\hline $\begin{array}{c}\text { Candidats } 1913 \\
\left(2^{\mathrm{e}} \text { tour }\right)\end{array}$ & Républicain libéral & $\begin{array}{c}\text { Républicain } \\
\text { démocrate }\end{array}$ & $\begin{array}{c}\text { Radical- } \\
\text { Socialiste }\end{array}$ \\
\hline Scores & $1235(20,6 \%)$ & $2239(37,4 \%)$ & $423(7,1 \%)$ \\
\hline
\end{tabular}

On peut tirer d'importantes conclusions des tableaux précédents. En présence d'un duel libéral-socialiste en 1910, l'électorat de gauche a préféré voter socialiste, cependant qu'un certain nombre d'électeurs de centregauche refusait cette stratégie. Au premier tour de 1913, on observe que le socialiste Le Gall a fidélisé 187 électeurs. De son côté, Salahun a récupéré un nombre équivalent de suffrages d'une gauche hostile au socialisme, de type Louis Hémon. À droite, l'électorat Villiers s'est scindé en deux au premier tour, de façon à peu près égale, le républicain démocrate prenant l'avantage grâce à l'appui d'abstentionnistes de 1910, catholiques modérés à l'évidence (politiquement), incapables de voter libéral en tous les cas. Le second tour de 1913 a vu s'opérer des recompositions importantes. La participation était en hausse. Le candidat libéral consolidait ses positions (+ 0,8 points). Salahun régressait : alors que les deux candidats de gauche avait groupé $14,5 \%$ des inscrits au premier tour, il n'en obtenait que la moitié (7,1 \%). Son maintien au second tour avait été décidé à la préfecture afin de favoriser dans la mesure du possible la victoire de Gaston de l'Hôpital. Ce dernier représentait en effet dans l'élection une droite traditionnelle, clairement identifiable et par conséquent un adversaire plus facile à combattre qu'un démocrate-chrétien. Ce calcul fut déjoué. Car, regrettait le préfet, " les combinaisons à longue échéance, l'électeur ne les conçoit pas ". Un bon nombre de républicains modérés (centre-gauche) s'étaient reportés sur Paul Simon.

La circonscription de Carhaix offre également un cas exemplaire. Quatre candidats se disputaient les suffrages au premier tour : le clérical-réactionnaire Jacques Corbel, le socialiste Le Tréis et deux radicaux, le maire de Carhaix Ferdinand Lancien et le conseiller général de Huelgoat Fégean. Au second tour, la droite présenta un démocrate-chrétien, Le Goasguen.

\section{Tableau 11 - Résultats des élections législatives dans la circonscription de Châteaulin II en 1914 (en nombre de suffrages exprimés)}

Premier tour

\begin{tabular}{|c|c|c|c|}
\hline Inscrits & Abstentions & Jacques Corbel & Total de la gauche \\
\hline 15491 & 3968 & 2317 & 9092 \\
\hline \multicolumn{4}{|l|}{ Second tour } \\
\hline Inscrits & Abstentions & Henri Le Goasguen & Ferdinand Lancien \\
\hline 15490 & 4180 & 3860 & 7072 \\
\hline
\end{tabular}


Le tableau 11 est très instructif. Il met en évidence une érosion nette des voix de gauche au second tour. Cela atteste l'existence d'un électorat modéré non clérical qui s'est reporté sur le démocrate-chrétien Le Goasguen après avoir refusé la candidature d'un réactionnaire et voté à gauche au premier tour. L'existence d'une telle sensibilité avait déjà été mise en évidence au début de la Troisième République. On se souvient qu'une large part des abstentionnistes de février 1871 dans la circonscription de Carhaix avait opté en 1876 pour le modéré Joseph Nédellec, opposé au royaliste ultra Henri de Legge.

L'examen des reports lors d'un second tour renseigne donc sur les identités et sensibilités politiques de l'électorat. On peut également individualiser ce courant dans la circonscription morbihannaise de Gourin.

\section{L'exemple de la circonscription de Gourin avant-guerre}

La circonscription de Pontivy II (Guéméné, Gourin, Le Faouët) présente un cas unique d'alternance politique régulière : en 1893 et 1898, elle votait à gauche, en 1902 et en 1906 à droite, en 1910 à gauche, en 1914 à droite.

Tableau 12 - Résultats des élections législatives dans la circonscription de Gourin (en suffrages pour le second tour de 1902, 1906, 1910 et 1914)

\begin{tabular}{|c|c|c|c|c|c|}
\hline & Inscrits & Abstentions & Droite & Gauche & Total \\
\hline $1902(2)$ & 11939 & $1986(16,6 \%)$ & 5652 & 4317 & 9969 \\
1906 & 12858 & $2178(16,9 \%)$ & 5559 & 5092 & 10651 \\
1910 & 13287 & $2708(20,4 \%)$ & 5034 & 5475 & 10509 \\
1914 & 13525 & $2898(21,4 \%)$ & 5524 & 4998 & 10522 \\
\hline
\end{tabular}

Sur les trois derniers scrutins, on constate que le nombre de votants a été quasiment identique $(=10500)$, inscrits et abstentionnistes augmentant parallèlement ${ }^{10}$. Le tableau 13 simplifie ces résultats.

Tableau 13

\begin{tabular}{|c|c|c|c|c|}
\hline & Inscrits & Abstentions & Droite & Gauche \\
\hline $1902(2)$ & 12000 & 2000 & 5700 & 4300 \\
1906 & 12800 & 2200 & 5500 & 5100 \\
1910 & 13300 & 2700 & 5000 & 5500 \\
1914 & 13500 & 2900 & 5500 & 5000 \\
\hline
\end{tabular}

Sur les trois derniers scrutins de la Troisième République, on observe que le socle de droite, c'est-à-dire le nombre minimum d'électeurs, en apparence incompressible, est de 5000 voix (1910), tout comme le socle de la

10. L'accroissement de l'abstention en 1910 et 1914 est dû à la retombée de l'agitation de la question religieuse. 
gauche (1914). Cela nous donne entre 500 et 600 électeurs " centraux ", soit un peu moins de $5 \%$ du corps électoral. C'est le choix de ces électeurs " centraux " qui détermine la majorité. En 1906, ils optèrent à 80 \% pour la droite, permettant à Guy de Boissieu d'être réélu avec 52,19 \% des voix. En 1910, la plupart se reportèrent à gauche. Alfred Brard l'emporta avec 52,09 \% des voix. En 1914, ses prises de position inquiétèrent cet électorat " central " : Victor Robic obtint 51,98 \% des suffrages.

En 1914, la droite avait désigné, après beaucoup d'hésitations, le maire du Faouët Victor Robic. Sa personnalité était de nature à séduire le centregauche. Il avait en effet été silloniste. Qui plus est, l'évolution politique de Brard vers le radicalisme l'avait coupé des catholiques modérés, à qui il devait en grande partie son siège.

"Les réactionnaires et les cléricaux ont exploité contre le député sortant le dépôt de son projet de loi sur l'autorisation préalable à l'ouverture des écoles libres dans les communes de moins de 3000 habitants. Ils lui ont fait grief d'avoir trahi le mandat dont il était investi de la part des catholiques fidèlement attachés à leurs traditions en s'inféodant au parti radicalsocialiste. Ils lui ont reproché comme un crime de lèse-Patrie ses mots contre la loi des trois ans."

Aux yeux du sous-préfet de Pontivy, il n'y avait rien là qui soit de nature à diminuer le crédit du député. Pourtant, force est de constater que la campagne aboutit à un déplacement suffisant de voix pour le mettre en échec et faire le succès de Robic.

On dispose d'une intéressante lettre du maire de Saint-Caradec, dans le canton de Guéméné. L'élu, républicain, y analysait les causes de l'échec. Elles étaient au nombre de trois. Il y a d'abord l'impact du retour à une laïcité militante après la démission d'Aristide Briand en novembre 1910. Robic sut en outre élargir sa base électorale en s'attachant des électeurs des classes moyennes qui avaient soutenu Brard en 1910 mais qu'alarmaient les augmentations d'impôt présentes et celles dont on les menaçait. La législation sociale des gouvernements radicaux serait-elle suffisante pour contrebalancer ces désertions parmi les couches populaires? En prenant l'exemple de la loi sur les familles nombreuses, le maire de Saint-Caradec dénonçait au lendemain du scrutin des mesures électoralement inefficaces. En effet, " ceux qui en bénéficient n'ont pas donné une voix de plus à la République [tandis que] beaucoup se sont retirés de la lutte ", en particulier des propriétaires indépendants qui voyaient que désormais leurs journaliers vivaient mieux qu'eux-mêmes ${ }^{11}$. Il est évident que ces classes moyennes constituaient pour partie l'électorat central. Suivant qu'il inclinait à droite ou à gauche, la victoire changeait de camp. Victor Robic avait su séduire l'électorat modéré, impressionné par son catholicisme ardent et rassuré par son passé silloniste. Il rejoignit en fait la Fédération Républicaine, un groupement politique constitué par les progressistes mélinistes. Ce groupement se situait alors au centre-droit.

11. Arch. dép. du Morbihan, M 4719, lettre du maire de Saint-Caradec, 29 avril 1914. 


\section{Une nécessité tactique : l'élection se gagne au centre}

La meilleure preuve de ce rôle capital du centre en politique, c'est-àdire des modérés, est donnée dans la circonscription de Pontivy. Il peut paraître surprenant de choisir comme exemple une circonscription qui fut le fief électoral du président du groupe parlementaire des Droites (monarchistes) au Parlement, le comte Paul de Lanjuinais. C'est un lieu commun dans les rapports contemporains de dire que Lanjuinais ne devait sa victoire qu'aux flots d'argent dont son immense fortune lui permettait d'arroser sa circonscription. Deux raisons essentielles expliquaient en fait les réélections de Paul de Lanjuinais : d'une part, les divisions internes aux républicains, d'autre part l'incapacité où ils se sont trouvés de fidéliser les électeurs du centre.

\section{La course au centre pour la gauche : les élections législatives de 1889 à 1898}

Le parti républicain de la circonscription de Pontivy sut en dix ans considérablement élargir ses positions. En 1889, Paul de Lanjuinais remporta le scrutin avec une avance de plus de 3000 voix sur son adversaire, le radical Charles Fagot. Il apparaissait clairement qu'un candidat trop marqué à gauche serait incapable de rassembler les voix des campagnes aux législatives. Les républicains désignèrent donc en 1893 un notable plus modéré Jean Robo. À la veille du scrutin, le receveur des contributions indirectes à Baud écrivait que «Mr Robo pourrait obtenir de bons résultats en étant bien secondé [car] le campagnard paraîtrait assez disposé à abandonner le candidat réactionnaire ${ }^{12} "$. Entre les deux élections, le nombre des inscrits, comme celui des votants, était quasiment identique, soit 12500 votants pour 16000 inscrits. Il s'agissait donc probablement des mêmes électeurs, à peu de choses près. Robo ramena l'écart avec Lanjuinais à 1300 voix. Il avait séduit les électeurs les plus modérés de la droite, ceux qui rejetaient le royalisme de Lanjuinais mais que la question religieuse empêchait jusque-là de voter à gauche. Le choix d'un nouveau candidat en 1898 confirma la stratégie républicaine de séduction prioritaire du centre. Leur nouveau candidat fut le chirurgien Pierre-Charles Langlais, un catholique pratiquant, rallié à la République, et dénoncé comme clérical par ses adversaires radicaux. Il avait repris la municipalité de Pontivy aux anticléricaux en 1896. Il avait organisé l'accueil du président de la République Félix Faure en août 1896. Le retentissement de l'événement avait certainement contribué à renforcer son assise. Sa profession de foi était extrêmement modérée. Il obtint 50,10 \% des suffrages exprimés. La circonscription de Pontivy basculait à gauche.

12. Arch. dép. du Morbihan, M 4713, rapport du receveur des contributions indirectes à Baud, 14 août 1893. 


\section{L'électorat «central » repasse à droite : les élections législatives de 1899 à 1910}

La fragile victoire de Langlais fut très contestée par la droite. Ne souhaitant pas que son élection soit entachée du moindre soupçon, le député démissionna et remit son mandat en jeu. Pour ne pas risquer une primaire au premier tour, Langlais avait en effet inconsidérément multiplié les concessions aux radicaux. Le sous-préfet s'en désolait :

" On craint que les quelques timides qui, aux élections dernières, ont voté pour le candidat républicain, ceux qui, en présence du succès obtenu, n'attendaient qu'une occasion pour venir au gouvernement, ne fassent un mouvement de recul en face de ce programme qui les atteint dans leur foi religieuse ${ }^{13}$."

Paul de Lanjuinais l'emporta avec 3000 voix d'avance. Langlais avait perdu au centre, du fait de concessions de programme faites aux radicaux. Il n'avait pas récupéré à gauche ces pertes car les électeurs anticléricaux avaient été ulcérés par plusieurs de ses votes parlementaires, en parfaite communion avec ceux de la droite.

En 1910, le docteur Langlais reçut une nouvelle fois l'investiture du parti républicain. Il se lançait sous les couleurs de l'Alliance Républicaine Démocratique (ARD), c'est-à-dire ce groupement constitué des progressistes qui avaient soutenu le gouvernement Waldeck-Rousseau. Ses rivaux radicaux apportèrent leur soutien, y compris financier, à la candidature du socialisant Abel Craissac. À eux deux, les candidats de gauche capitalisaient 6700 suffrages, l'avantage revenant nettement au républicain modéré (6400 voix). Lanjuinais l'emporta finalement avec 700 voix d'avance. Les républicains avaient cependant repris espoir. L'état de santé du président du Conseil général (Lanjuinais était quasiment paralysé) leur laissait espérer de prochaines opportunités. En 1910, seule la conviction que la circonscription passerait à gauche s'il se retirait l'avait convaincu de repartir. En 1914, il s'effaça devant son gendre, Arthur de la Villesboisnet, conseiller général de Loire-Inférieure et richissime spéculateur en Bourse.

\section{La circonscription de Pontivy en 1914 : équilibre politique et légère majorité à droite}

Arthur de La Villesboisnet fut le candidat de la Fédération des Républicains Indépendants (FRD), c'est-à-dire des libéraux. Elle était née en 1911, représentait la droite non monarchiste et était dirigée par le député de Hennebont Ernest Lamy et par le sénateur Jean Guilloteaux. La FRI donna sans rechigner en 1914 son investiture à Arthur de La Villesboisnet car il était plus modéré que son beau-père. Il était tout à fait représentatif de la modernisation de la droite et, par exemple ses meetings se faisaient au chant de La Marseillaise. Son adversaire fut Paul Maulion, conseiller général de Mauron, dans l'arrondissement de Ploërmel. Richissime avocat,

13. Arch. dép. du Morbihan, M 4715, rapport du sous-préfet de Pontivy, 6 janvier 1899. 
au talent oratoire éprouvé, il se présentait sous la double étiquette radicale-socialiste et de l'ARD, force politique qu'il faut situer au centre-gauche. Il pouvait ainsi espérer maintenir l'union de la gauche autour de sa personne et enfin grouper une majorité de gauche sur la circonscription. Il semble en fait que son étiquette de radical ait affolé une partie des électeurs modérés. Le sous-préfet relatait :

" J'ai entendu plusieurs républicains modérés de Pontivy, notamment des commerçants, faire grief à Monsieur Maulion d'être à la fois inscrit comme membre du Comité exécutif du parti radical-socialiste de la rue de Valois et comme membre de l'Alliance Démocratique. Ils estiment que cette affiliation à deux associations à idées très différentes, provoquera des abstentions parmi les républicains modérés. Ces derniers disent qu'après les récents scandales éclaboussant le parti radical-socialiste en la personne de ses chefs, un candidat honnête ne peut pas se recommander de ce parti ${ }^{14}$."

La crainte des élites républicaines modérées s'avéra fondée. Dans quelle mesure la double affiliation de Maulion lui fit-elle du tort? Il est impossible de le déterminer exactement. Il est néanmoins certain qu'elle dissuada de voter à gauche un pourcentage suffisant d'anciens électeurs Lanjuinais de sensibilité modérée. En face, Arthur de la Villesboisnet, à qui le soutien de son beau-père garantissait les voix de la droite dure, tâchait en revanche de courtiser le centre en se réclamant de la République la plus libérale. Il l'emporta avec 50,06 \%. C'est dire que la victoire avait tenu à un très faible déplacement de voix. On prêta à La Villeboisnet l'intention de s'inscrire au groupe parlementaire de l'ARD (centre-gauche), groupement dont il était depuis plusieurs années adhérent. Il rejoignit en définitive les non-inscrits.

Dans plusieurs circonscriptions du Morbihan, les élections s'étaient jouées au centre, à quelques centaines de voix près, parfois moins. On peut en fait généraliser un jugement du sous-préfet de Pontivy pour définir le caractère politique des électeurs de la circonscription de Gourin : «Dans un milieu où la versatilité du suffrage universel est inhérente à la nature des choses sociales, l'instabilité du siège doit être inspiratrice pour le député de la prudence la plus sage et la plus circonspecte. Le milieu est très impressionnable, très particulariste, assez hostile aux innovations parce que d'un conservatisme politique et religieux modéré mais toutefois animé d'un esprit de libéralisme assez tolérant ${ }^{15}$. " Cette versatilité apparente tenait en fait à la recherche par l'électorat central du candidat le plus modéré possible.

\section{Le rôle de l'électorat modéré dans le Finistère}

La comparaison des scrutins atteste l'existence dans la circonscription de Pont-l'Abbé d'un électorat de gauche modérée. En 1906, dans le contexte des inventaires et compte-tenu de la personnalité du candidat Georges Le Bail, radical anticlérical, ces électeurs optèrent pour le candidat de droite,

14. Arch. dép. du Morbihan, M 4719, rapport du sous-préfet de Pontivy, 27 mars 1914. 15. Arch. dép. du Morbihan, M 4719, rapport du sous-préfet de Pontivy, 30 avril 1914. 
Henri de Servigny. La désertion de la circonscription par Le Bail en 1910 et la promotion du plus mesuré Édouard Plouzané amena le déplacement d'un certain nombre de voix modérées vers la gauche. En 1914 à l'inverse, le parachutage du fils de l'ancien député, Georges Le Bail-Maignan, rejeta ces électeurs à droite. Cette " versatilité du suffrage universel " pointée par le sous-préfet de Pontivy, même si dans le cas de Pont-l'Abbé elle était insuffisante pour faire basculer la circonscription, tenait là encore au positionnement d'une frange assez limitée d'électeurs, les modérés, ces électeurs du " centre ". On observe que cet électorat était assez important à Loctudy. $\mathrm{Sa}$ " versatilité " explique les changements de majorités qu'on y observera entre les deux guerres.

Tableau 14 - Les votes de l'électorat modéré en 1906 et au second tour de 1910 et 1914 dans la circonscription de Pont-L'Abbé

(en valeur absolue et relative)

\begin{tabular}{|c|c|c|c|c|}
\hline & Inscrits & Abstentions & Gauche & Droite \\
\hline 1906 & 13052 & $2312(17,7 \%)$ & $5213(39,9 \%)$ & $5575(42,7 \%)$ \\
1910 & 13959 & $3011(21,6 \%)$ & $6055(43,4 \%)$ & $4661(33,4 \%)$ \\
1914 & 14441 & $3086(21,4 \%)$ & $5679(39,3 \%)$ & $5557(38,5 \%)$ \\
\hline
\end{tabular}

Tableau 15 - Les votes de l'électorat modéré en 1906 et au second tour de 1910 et 1914 à Loctudy (en valeur absolue et relative)

\begin{tabular}{|c|c|c|c|c|}
\hline & Inscrits & Abstentions & Gauche & Droite \\
\hline 1906 & 663 & $96(14,5 \%)$ & $164(24,7 \%)$ & $403(60,8 \%)$ \\
1910 & 735 & $120(16,3 \%)$ & $338(46,0 \%)$ & $277(37,7 \%)$ \\
1914 & 738 & $127(17,2 \%)$ & $257(34,8 \%)$ & $354(48,0 \%)$ \\
\hline
\end{tabular}

Le poids de l'électorat modéré peut également être mis en évidence dans le Léon. La circonscription de Saint-Pol-de-Léon a été le fief électoral du légitimiste Émile de Kermenguy (1876-1893) puis d'Albert de Mun après 1894.

Dans le canton de Plouzévédé, on constate l'affaiblissement continu de la gauche jusqu'en 1889, une fraction de l'électorat se voyant contrainte de voter à droite. Le score de l'adversaire républicain d'Albert de Mun en 1894 résulte du report massif d'électeurs royalistes sur son nom. Il ne signifie donc rien en lui-même. Le score de 1914 mérite en revanche quelques explications.

Tableau 16 - Comparaison des scores des candidats de gauche entre 1910 et 1914 (dans trois cantons de la circonscription de Saint-Pol en valeur relative sur les inscrits)

\begin{tabular}{|c|c|c|c|}
\hline & Inscrits 1910/1914 & Score Le Morvan 1910 & Score Caill 1914 \\
\hline Plouzévédé & $3649 / 3705$ & 742 voix $(20,3 \%)$ & 1515 voix $(40,9 \%)$ \\
Taulé & $3024 / 3082$ & 497 voix $(16,4 \%)$ & 1067 voix $(34,6 \%)$ \\
Landivisiau & $4178 / 4151$ & 938 voix $(22,5 \%)$ & 1433 voix $(34,5 \%)$ \\
\hline
\end{tabular}


L'amélioration du score de la gauche en 1914 est à mettre en relation avec la désignation d'un modéré comme candidat. En 1910, la gauche avait choisi pour porter ses couleurs un ardent radical, farouche anticlérical Yves Le Morvan. Rassembler dans ces conditions près du tiers des votants de la circonscription léonarde était déjà en soi un succès. Mais ses opinions trop arrêtées avaient désespéré les électeurs du centre. La gauche eut l'habileté en 1914 de donner l'investiture à une des étoiles montantes de la nouvelle génération républicaine, le vétérinaire de Plouzévédé, Yves Caill. Âgé de 33 ans, maire de sa commune, d'opinions très modérées, il était le candidat idéal, seul susceptible de séduire l'électorat de centre-droit. L'évolution nationaliste d'Albert de Mun éveillait en effet chez lui de nombreuses réticences.

Albert de Mun redoutait de voir " passer à gauche " l'électorat modéré de sa circonscription, les démocrates-chrétiens particulièrement. La querelle religieuse avait retardé chez ces électeurs modérés la prise de conscience qu'ils avaient de plus en plus d'affinités avec les républicains de gauche. Le durcissement de la droite facilita cette prise de conscience. L'existence d'une sensibilité modérée, un centre-droit susceptible de voter peut-être un jour pour le centre-gauche, était du reste attestée dès 1905.

" La deuxième circonscription de Morlaix appartient au pays très spécial du Léon. Les grands propriétaires terriens et avant tout le clergé sont les deux grandes forces qui dirigent une population rurale passive. En face d'eux se meut une force relativement nouvelle, les julots, paysans riches du Léon, véritable aristocratie campagnarde qui constitue une caste tout aussi fermée que l'ancienne noblesse. Les julots, soumis au clergé comme tous les Léonais, ont en général des tendances républicaines et formeront peutêtre un jour le noyau d'un parti républicain ${ }^{16}$."

En 1914, même si cet apport fut insuffisant pour mettre en échec Albert de Mun, Yves Caill sut séduire l'électorat modéré, démocrate-chrétien en partie, catholique et républicain, éloigné des positions tranchées en tous les cas.

Qu'est-ce que le centre en politique? Sans doute existe-t-il plusieurs centres, un centre-droit et un centre-gauche au moins, se rejoignant parfois, divergeant souvent en fonction des enjeux et des personnalités en cause. Deux caractéristiques cependant émergent : d'une part le rejet de tout extrême, d'autre part l'impossibilité consécutive à respecter une discipline, d'où une apparence de versatilité, d'instabilité. Cet électorat du centre, qu'on a dit " central ", amalgame différents éléments. Il est, pour une part, l'héritier d'une tradition de républicanisme catholique. En ce sens, il a toujours témoigné, jusque dans les périodes de tension extrême, que les deux notions ne sont absolument pas incompatibles. Il découle aussi de l'appel au ralliement du pape Léon XIII. Sur ce point, une différence doit être faite. D'un côté, on a ceux qui, les premiers, obéirent au pape, ces libé-

16. Arch. dép. du Finistère, 3M 300, rapport du préfet, 10 octobre 1905. 
raux peu à peu absorbés par les conservateurs, et qui, d'un hypothétique centre-droit, évoluèrent bientôt vers la droite. Albert de Mun en fournit l'archétype. De l'autre, on a ces démocrates-chrétiens dont Hippolyte Gayraud fut le symbole et dont le Sillon prolongea un temps l'héritage. L'expression électorale du centre est étroitement soumise à divers types de conditions qui en rendent la saisie difficile. La prégnance du scrutin majoritaire est un handicap majeur. La division consécutive à sa double filiation gêne en outre toute affirmation électorale autonome.

Pourtant, l'électorat central existe toujours. Ses aspirations sont les mêmes qu'hier. Peut-il survivre à une tentative d'institutionnalisation? Beaucoup, historiquement, ont tenté le pari de créer cette force " centrale " sur l'échiquier politique, ce parti qu'on pourra, à ce point de notre raisonnement, dire " centriste ". L'incapacité d'un parti centriste à rassembler tous les éléments disparates de l'électorat central témoigne de son inadaptation aux cadres imposés par le scrutin majoritaire. Le centre peut-il être dans ces conditions autre chose qu'une sensibilité politique? Mais est-ce déjà si négligeable de rester, dans l'opinion, cet appoint nécessaire à toute majorité et, sans que quiconque cherche illusoirement à l'incarner, de demeurer tout simplement l'aiguillon qui préserve des excès? 


\section{RESUME}

L'étude de la vie politique en Bretagne sous la Troisième République révèle le poids crucial lors des scrutins d'un électorat modéré qui ne s'est jamais reconnu dans le face-à-face des royalistes et des républicains. Cette personnalité politique originale se manifeste par une apparente instabilité électorale. En réalité, ces électeurs faisaient alterner vote à gauche et vote à droite avec pour souci constant de favoriser le candidat le moins " marqué " politiquement.

Ce comportement se remarque dès 1871. L'importance de cet électorat au centre de l'échiquier politique a dès lors conduit les états-majors à adapter leurs stratégies pour gagner les élections.

Si les élections sénatoriales permettent d'observer la première mise en place d'une conjonction des centres, les stratégies déployées lors des législatives montrèrent de plus en plus à quel point c'était bien au centre de l'échiquier que faisait la décision politique.

Dans le Finistère, avant la Première Guerre Mondiale, les candidats sillonistes, héritiers de l'aile gauche de la démocratie-chrétienne, incarnèrent parfaitement ce " centre " à la jonction de la droite et de la gauche. Leur évolution politique future a cependant prouvé la difficulté d'une expression autonome d'une sensibilité politique authentiquement centriste.

\section{ABSTRACT}

Studying political life in Brittany under the Third Republic reveals the crucial importance at election-time of a moderate electorate, which never felt akin to the traditional face-à-face between Royalists end Republicans. Such an original political personality is manifest through apparent electoral instability. As a matter of fact, these voters alternated the left vote with the right vote showing constant determination to favour candidates with the least apparent political bias.

Such behaviour was evident as early as 1871. The significance of that electorate at the center of the political scene thus led party officials to adapt their strategies so as to win elections.

Although the first establishment of some kind of centre conjunction may be observed through senatorial elections, the strategies displayed at legislative elections gradually showed that decisions were actually made at the very center of the political scene.

In Finistère before the First World War, the candidates of the Sillon Movement - the heirs of the Christian Democrats' left wing - truly embodied that centre located at the junction between right and left wings. However, their future political evolution was to show how difficult was the independent expression of a political trend possessing genuinely centre-biassed sympathy. 